\title{
Role of GntR Family Regulatory Gene SCO1678 in Gluconate Metabolism in Streptomyces coelicolor M145
}

\author{
Olga Tsypik, ${ }^{1,2}$ Roman Makitrynskyy, ${ }^{1}$ Agnieszka Bera, ${ }^{2}$ Lijiang Song, ${ }^{3}$ \\ Wolfgang Wohlleben, ${ }^{2}$ Victor Fedorenko, ${ }^{1}$ and Bohdan Ostash $^{1}$ \\ ${ }^{1}$ Department of Genetics and Biotechnology, Ivan Franko National University of Lviv, Lviv 79005, Ukraine \\ ${ }^{2}$ Microbiology and Biotechnology, Interfaculty Institute of Microbiology and Infection Medicine, University of Tübingen, \\ Tübingen, Germany \\ ${ }^{3}$ Department of Chemistry, University of Warwick, Coventry CV4 7AL, UK
}

Correspondence should be addressed to Bohdan Ostash; b.ostash@lnu.edu.ua

Received 2 January 2017; Revised 7 March 2017; Accepted 26 March 2017; Published 27 April 2017

Academic Editor: Pengjun Shi

Copyright (C) 2017 Olga Tsypik et al. This is an open access article distributed under the Creative Commons Attribution License, which permits unrestricted use, distribution, and reproduction in any medium, provided the original work is properly cited.

\begin{abstract}
Here we report functional characterization of the Streptomyces coelicolor M145 gene SCO1678, which encodes a GntR-like regulator of the FadR subfamily. Bioinformatic analysis suggested that SCO1678 is part of putative operon (gnt) involved in gluconate metabolism. Combining the results of SCO1678 knockout, transcriptional analysis of gnt operon, and Sco1678 protein-DNA electromobility shift assays, we established that Scol678 protein controls the gluconate operon. It does so via repression of its transcription from a single promoter located between genes SCO1678 and SCO1679. The knockout also influenced, in a mediumdependent manner, the production of secondary metabolites by $S$. coelicolor. In comparison to the wild type, on gluconatecontaining minimal medium, the SCO1678 mutant produced much less actinorhodin and accumulated a yellow-colored pigment, likely to be the cryptic polyketide coelimycin. Possible links between gluconate metabolism and antibiotic production are discussed.
\end{abstract}

\section{Introduction}

Bacteria of genus Streptomyces are abundant soil dwellers having unparalleled capacity to produce bioactive small molecules and to assimilate complex plant (e.g., lignins and cellulose) and animal polymers [1]. These properties fuel interest in Streptomyces as a source of novel drug candidates [2], valuable hydrolytic enzymes [3], and ecofriendly plant protection strategies [4]. Streptomycetes have evolved a complicated regulatory network that coordinates their primary metabolism with biosynthetic pathways responsible for the production of specialized secondary metabolites and breakdown of polymers $[5,6]$. Core regulators of primary metabolism of streptomycetes are intimately linked to antibiotic production [7]. Streptomyces genomes are also very large, between 6 and $11 \mathrm{Mbp}$, and harbor a lot of regulatory genes. The overall understanding of how these regulatory genes control transcription is still unclear [8]. This limits our current ability to take full advantage of genomic potential of Streptomyces for its biomedical and industrial applications. Therefore, it is important to continue functional characterization of various regulatory genes in well-known model species, like the best studied Streptomyces coelicolor A3(2) (or its derivative M145).

Recently we have carried out extensive in silico analysis of GntR family transcriptional factors, one of the biggest and yet poorly understood groups of regulators in Streptomyces [9]. As a result, twelve GntR regulators have been described that are conserved across the Streptomycetaceae family. We refer to them as "core GntRs" to highlight their potential important function in Streptomyces biology. Two of them, WhiH and DasR, have been extensively studied in the past. WhiH controls early steps of sporulation $[10,11]$, while DasR is a pleiotropic regulator of multiple carbohydrate transporters, chitin metabolism and antibiotic production genes [12]. A third regulator, Sco0823, was recently proposed by us to participate in ferric ion uptake [9]. Functions of the nine other core GntRs are yet to be explored. Here we report functional 
TABLE 1: Bacterial strains and plasmids used in this work.

\begin{tabular}{|c|c|c|}
\hline Strains or plasmids & Description & Source or reference \\
\hline S. coelicolor M145 & $\mathrm{SCP}^{-}, \mathrm{SCP}^{-}$derivative of $\mathrm{A} 3(2)$; ACT and RED producer & [15] \\
\hline S. coelicolor $\Delta \mathrm{gntR}$ & SCO1678 knockout in M145 & This work \\
\hline S. coelicolor pKC-SCO1678 & SCO1678 overexpression in M145 & This work \\
\hline S. coelicolor $\mathrm{pKC1139}$ & M145 with pKC1139 empty vector & This work \\
\hline S. coelicolor pGUS & M145 carrying pGUS & This work \\
\hline S. coelicolor pGUS-gntRp & M145 carrying pGUS-gntRp & This work \\
\hline Escherichia coli $\mathrm{DH} 5 \alpha$ & Routine cloning host & Life Technologies \\
\hline E. coli ET12567 (pUZ8002) & Host for conjugative DNA transfer & [13] \\
\hline E. coli BW25113 (pIJ790) & Host for recombineering experiments & [13] \\
\hline E. coli BL21 (DE3) GOLD & Strain for recombinant protein production & Stratagene \\
\hline pIJ790 & ts-plasmid carrying genes for $\lambda$-RED recombination, $\mathrm{CmlR}$ & [13] \\
\hline pLeere & Carrying $\operatorname{acc}(3) I V$ flanked by loxP-sites, apramycin and ampicillin resistances & Luzhetskyy \\
\hline pKC1139 & pSG5 ts-replicon; apramycin-resistant (AmR) shuttle vector & [15] \\
\hline pKC-SCO1678 & pKC1139 harboring SCO1678 with promoter region; SCO1678 overexpression, AmR & This work \\
\hline pKC0702-SCO1678 & pKC0702 harboring SCO1678 with 3-kb flanking region, HygR & This work \\
\hline pKC0702-SCO1678::Am & SCO1678 knockout construct; $\Delta$ sco1678::hyg (HygR and AmR) & This work \\
\hline pGUS & Promoterless gusA-containing plasmid, apramycin resistance & [17] \\
\hline pGUS-gntRp & pGUS with gntRp-gusA fusion, AmR & This work \\
\hline pET28a & Protein expression vector, pET-system & Novagen \\
\hline pET28a-SCO1678 & Scol678-6His protein expression & This work \\
\hline
\end{tabular}

characterization of S. coelicolor M145 gene SCO1678, which encodes a GntR type regulator from the FadR subfamily. We demonstrate that SCO1678 encodes a repressor of the gluconate operon and that the promoter of gluconate kinase gene SCO1679 is the main target of Scol678 regulatory action. Interestingly, a knockout of SCO1678 also altered the secondary metabolite profile of $S$. coelicolor. Our work reveals one more regulatory checkpoint that links primary metabolism and antibiotic production in actinomycetes.

\section{Materials and Methods}

2.1. Bacterial Strains and Culture Conditions. Bacterial strains used in this work are listed in Table 1. E. coli DH5 $\alpha$, ET12567 (pUZ8002), and BW25113 (carrying pIJ790) were used for routine cloning, to perform intergeneric conjugation with Streptomyces species and to carry out RedETmediated gene replacement, respectively [13]. E. coli DH5 $\alpha$ and ET12567 (pUZ8002) were grown at $37^{\circ} \mathrm{C}$ in Luria-Bertani (LB) medium [14]; strain BW25113 was grown at $28^{\circ} \mathrm{C}$ in $2 \mathrm{x}$ YT medium (Tryptone: $20 \mathrm{~g}$, Yeast extract: $10 \mathrm{~g}$, and sodium chloride: $5 \mathrm{~g}$ per $1 \mathrm{l}$ of distilled water). All Streptomyces strains were grown at $28^{\circ} \mathrm{C}$. Solid SFM medium (soya flour, mannitol, agar-20 g/liter each) was used to harvest Streptomyces spores and to plate Streptomyces-E. coli matings. To analyze the gene transcription profile, precultures of Streptomyces strains were grown in TSB medium for $24 \mathrm{~h}$, and then mycelium was harvested by centrifugation, washed three times with water, and inoculated into SMM medium with $1 \%$ glucose or gluconate for $36 \mathrm{~h}$ [15]. To analyze antibiotic (actinorhodin (ACT), undecylprodigiosin (RED), and coelimycin (CPK)) production, liquid YMPG, R2YE, SMM, and Oxoid agar media, respectively, were used $[15,16]$. Where needed, media were supplemented with respective antibiotics.

2.2. DNA Techniques. Isolation of plasmid DNA from E. coli and chromosomal DNA from Streptomyces, DNA digestion by restriction endonucleases, agarose gel electrophoresis, and DNA ligation were performed using standard protocols $[14,15]$. E. coli transformation and intergenetic E. coliStreptomyces matings were performed as described in [15]. DNA amplification by PCR was generated with Taq (NEB) and Phusion (NEB) DNA polymerases. All plasmids were verified by DNA sequencing.

2.3. Construction of $p G U S-g n t R p$ Plasmid. Approximately 230-bp of the promoter region of $g n t R$ was amplified with primers SCO1678gusXbaI and SCO1678gusKpnI, digested, and cloned into XbaI and KpnI sites of pGUS-vector giving pGUS-gntRp. Oligonucleotides used throughout this work are listed in Table 2.

2.4. Construction of $p K C$-gntR Overexpression Plasmid and pSET-gntR for Complementation. SCO1678 (=gntR) coding sequence with 230 -bp promoter region was amplified with SCO1678 cmpl-f/SCO1678 cmpl-r primer pair. PCR product was digested with $\mathrm{BamHI}$ and $\mathrm{XbaI}$ and cloned into respective sites of moderate copy number vector pKC1139 and integrative plasmid pSET152, giving pKC-gntR and pSETgntR, respectively.

2.5. Construction and Verification of SCO1678 Knockout Strain. An in-frame deletion mutant $S$. coelicolor $\Delta \mathrm{gntR}$ was constructed using REDIRECT technology [13]. For this 


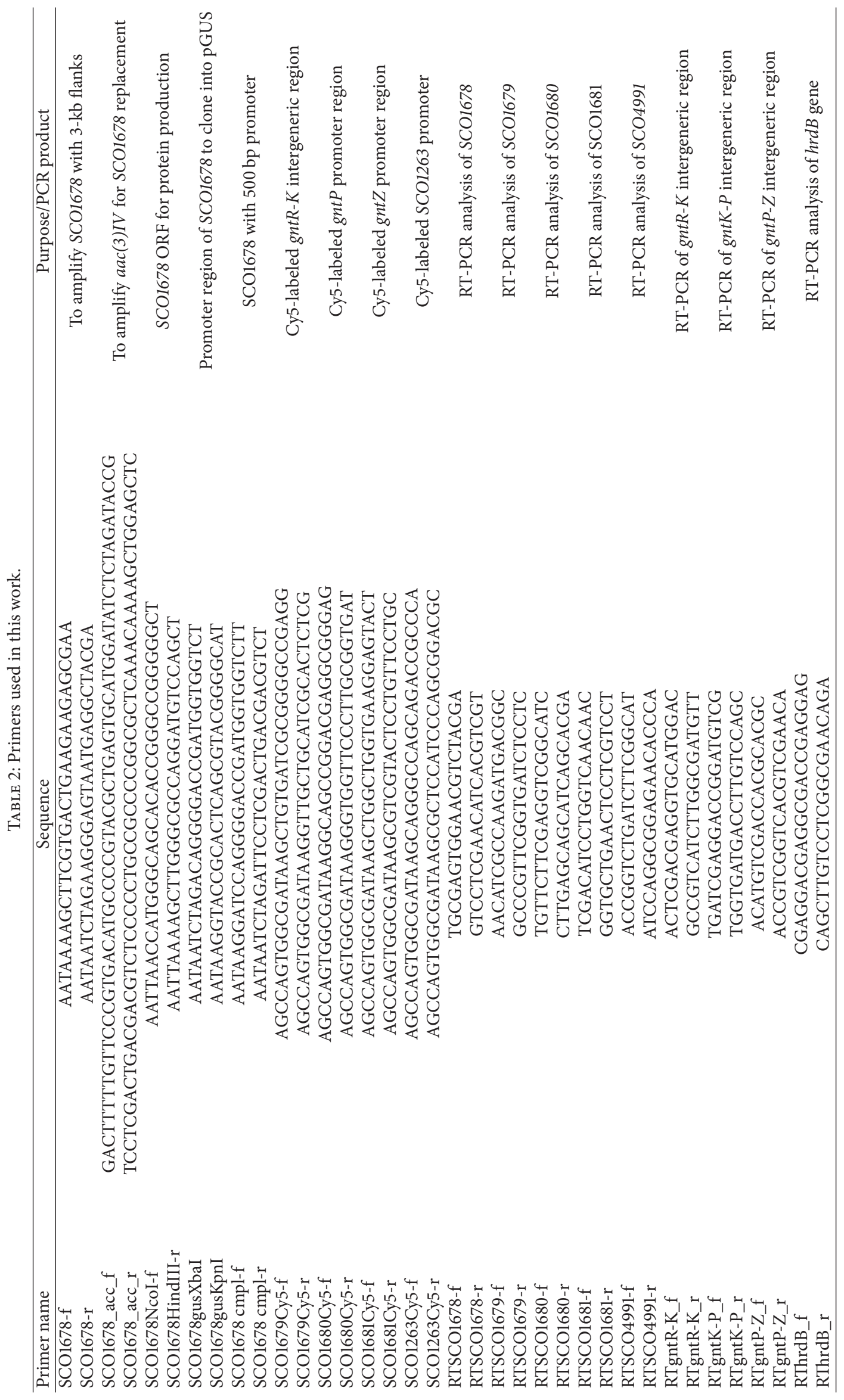


purpose, gene SCO1678 with 3-kb flanking regions was amplified from chromosomal DNA of $S$. coelicolor with the following primers: SCO1678-f and SCO1678-r. The PCR product was digested with HindIII and $\mathrm{XbaI}$ restriction endonucleases and subsequently cloned into respective sites of pKC0702. The obtained plasmid pKC0702-SCO1678 was transformed into $E$. coli BW25113 where replacement of SCO1678 by apramycin resistance cassette aac(3)IV was accomplished. The latter was amplified from plasmid pLeere using SCO1678_acc_f and SCO1678_acc_r primer pair. The knockout plasmid pKC0702-SCO1678::Am was introduced into the wild type $S$. coelicolor M145 followed by screening of apramycin-resistant and hygromycin-sensitive colonies. Positive clones were indicative that a double crossover had occurred between the homologous regions of the M145 genome and on the knockout construct. Markerless mutant $S$. coelicolor $\Delta$ gntR was generated by implication of sitespecific recombinase Cre as described in [18]. The SCO1678 disruption and marker eviction were confirmed via PCR (primers SCO1678 cmpl-f/SCO1678 cmpl-r).

2.6. RT-PCR. RNA for semiquantitative RT-PCR was isolated using RNeasy mini kit (Qiagen) according to recommendations of the supplier. RNA samples were checked for DNA contamination by PCR. For cDNA synthesis $3 \mu \mathrm{g}$ of total RNA was incubated with random primers for five minutes at $72^{\circ} \mathrm{C}$. The remaining components (RNase inhibitor, dNTPs, reverse transcriptase buffer, DTT, and ProtoScript II reverse transcriptase (NEB)) were subsequently added and reverse transcription (RT) was carried out at $42^{\circ} \mathrm{C}$ for $60 \mathrm{~min} .200 \mathrm{ng}$ of synthesized RT products was used as a template for subsequent PCR analysis with primers listed in Table 2. Obtained PCR products were separated on a 1.5\% agarose gel to analyze the transcription profile of genes of interest.

\subsection{Production and Purification of His-Tagged SCO1678 Pro-} tein. To produce C-terminally hexahistidine-tagged Sco1678 protein (GntR-His), its ORF was cloned into pET28a expression vector using PCR and primers SCO1678NcoI-f and SCO1678HindIII-r. Resulting plasmid was labeled as pET28aSCO1678. For GntR-His production, E. coli BL21 (DE3) GOLD carrying pET28a-SCO1678 was grown in LB supplemented with tetracycline and kanamycin until $\mathrm{OD}_{600}$ reached 0.5 ; then the culture was induced with $1 \mathrm{mM}$ IPTG (isopropylthiogalactoside) and incubated for six hours at $22^{\circ} \mathrm{C}$. Cells were collected by centrifugation and resuspended in a lysis buffer $\left(50 \mathrm{mM} \mathrm{Na} \mathrm{HPO}_{4}, 300 \mathrm{mM} \mathrm{NaCl}\right.$, and $20 \mathrm{mM}$ imidazole, $\mathrm{pH}$ 7) containing proteinase inhibitor (Roche). Cells lysis was achieved by two consecutive passages through a French press (American Instrument Corporation) at 1000 psi. The cell lysate was centrifuged at $18000 \mathrm{rpm}$ for 30 minutes and soluble fraction was applied to Ni-NTA agarose resin (Qiagen), washed two times with wash buffer $(50 \mathrm{mM}$ $\mathrm{Na}_{2} \mathrm{HPO}_{4}, 300 \mathrm{mM} \mathrm{NaCl}$, and $40 \mathrm{mM}$ imidazole, $\mathrm{pH}$ 7). The protein was eluted with $200 \mathrm{mM}$ imidazole and dialyzed against storage buffer $\left(50 \mathrm{mM} \mathrm{Na}_{2} \mathrm{HPO}_{4}, 300 \mathrm{mM} \mathrm{NaCl}\right.$, and $5 \%$ glycerol, $\mathrm{pH} 7$ ). Protein concentration was determined by Bradford assay.

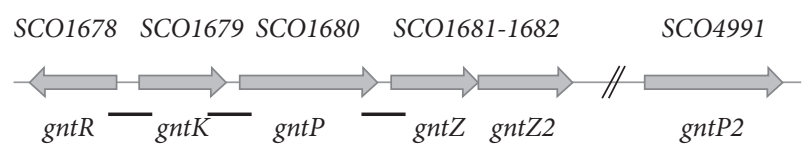

(a)

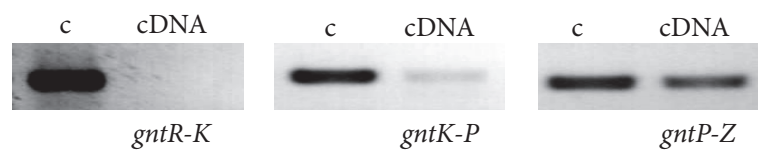

(b)

FIGURE 1: Genes for gluconate operon in S. coelicolor (a) and their transcriptional organization (b). Three black rectangles beneath the SCO genes indicate fragments amplified during RT-PCR analysis of intergenic regions (see (b)).

2.8. Electromobility Shift Assay (EMSA) of DNA-Protein Complexes. Putative promoter regions of SCO1263, gntP, gntZ, and $g n t R-K$ were amplified from chromosomal DNA of $S$. coelicolor with primers listed in Table 2 and subsequently labeled with indocarbocyanine (Cy5) as described in [19]. Cy5-labeled probes $(0.2 \mathrm{pmol})$ were incubated with purified recombinant GntR-His (see above) in concentrations of 0.93 to $4.18 \mathrm{pmol}$ in binding buffer $(20 \mathrm{mM}$ Tris/ $\mathrm{HCl} \mathrm{pH} 7.5$, $50 \mathrm{mM} \mathrm{KCl}, 10 \mathrm{mM} \mathrm{MgCl}_{2}, 5 \%$ (v/v) glycerol, and $0.5 \mathrm{mM}$ EDTA) for $25 \mathrm{~min}$ at $25^{\circ} \mathrm{C}$. Electrophoresis was carried out in $8 \%$ native polyacrylamide gel in $1 \mathrm{x}$ TBE buffer at $150 \mathrm{~V}$ for $60 \mathrm{~min}$. DNA bands were visualized by fluorescence imaging using a Typhoon Trio variable mode imager (GE Healthcare). EMSA was used to test whether gluconate, glucono-1,5lactone, and glucose are potential effector molecules for Sco1678 (at final concentration of $5 \mathrm{mM}$ in reaction mixture).

2.9. Analysis of $\beta$-Glucuronidase Activity. Strains carrying pGUS-gntR or pGUS plasmids were grown for $36 \mathrm{~h}$ in SMM supplemented with certain carbon source. Transcription level of $\beta$-glucuronidase from $g n t R$ promoter was examined according to [17].

2.10. Analysis of Antibiotic Production. ACT and RED production levels were quantified as described in [15], in YMPG and R2YE media, respectively. ACT production was analyzed in SMM medium with either glucose or gluconate as the sole carbon source.

\section{Results}

3.1. Analysis of gnt Operon in S. coelicolor. Gene SCO1678 encodes a 233 aa transcriptional factor from the GntR family of regulators. As a member of this family Scol678 protein consists of a N-terminal DNA-binding domain with GntRlike helix-turn-helix motif followed by a C-terminal effector binding/oligomerization domain. Based on the secondary structure of the C-terminal domain, Sco1678 was proposed to fall into the FadR subfamily of GntRs [9]. Divergently to $S C O 1678$, genes of putative gluconate ( $g n t)$ operon are located (Figure 1(a)). Gene SCO1679 ( $g n t K$ ) encodes gluconokinase that phosphorylates gluconate to glucono-5-phosphate which 


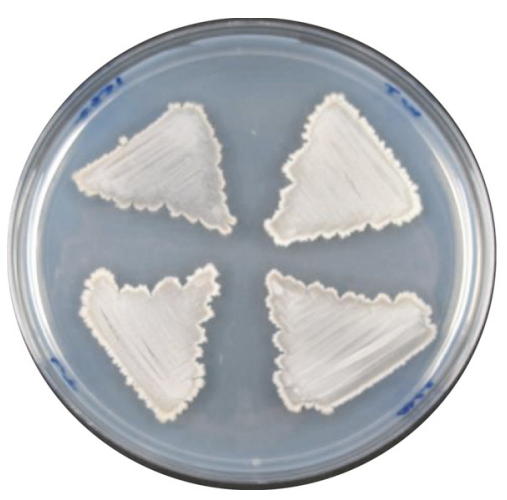

(a)
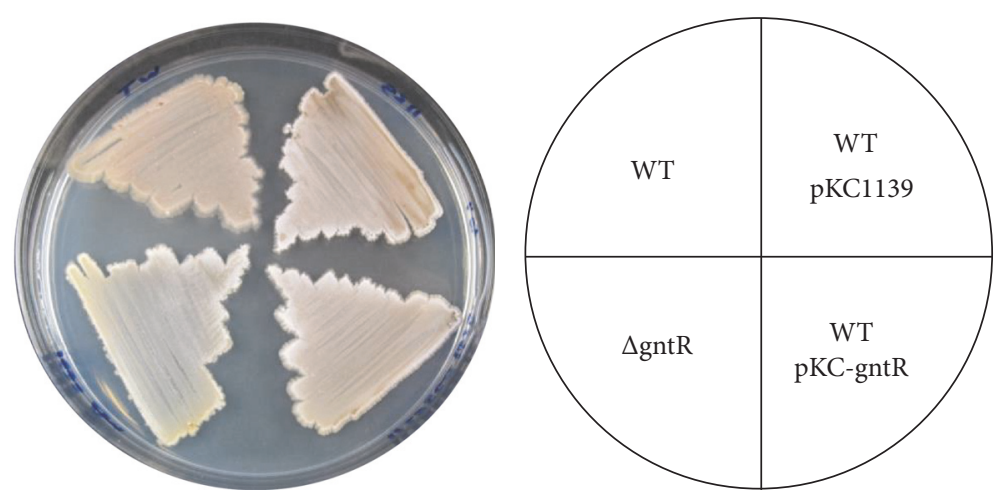

(b)

FIGURE 2: S. coelicolor strains grown on MM with glucose (a) or gluconate (b). WT: S. coelicolor M145; $\Delta$ gntR: S. coelicolor $\Delta$ gntR; WT pKCgntR: S. coelicolor harboring pKC-gntR; WT pKC1139: S. coelicolor plus empty vector pKC1139.

is then metabolized in the pentose phosphate pathway. Gene SCO1680 (gntP) encodes gluconate permease that transports the molecule into a cell. The next two genes, SCO1681SCO1682 (gntZ-gntZ2), overlap by 4 nucleotides and encode gluconate dehydrogenase and zinc-binding alcohol dehydrogenase, respectively. RT-PCR confirmed transcriptional coupling of genes SCO1679-1680-1681 (Figure 1(b)).

Another carbohydrate transport membrane protein Sco4991 shows 39\% identity to GntP permease of Bacillus subtilis and 38\% to S. coelicolor gluconate permease Scol680. The former, therefore, might be involved in gluconate uptake as well. This agrees with recent global analysis of the entire array of transport proteins in S. coelicolor, where Sco1680 and Sco4991 were annotated as high-affinity gluconate permease and gluconate permease, respectively [20].

3.2. Expression and Knockout of SCO1678. Organization and regulation of gnt operons in Escherichia coli and Bacillus subtilis are well studied [21, 22]. In both cases transcription of gnt genes is repressed by GntR, a protein that served as a prototype for the entire GntR family of regulators. From available in silico data we propose that SCO1678 also encodes GntR. If, contrary to our assumption, SCO1678 encodes an activator of gnt genes, then its deletion would lead to arrested or significantly reduced growth in presence of gluconate as the sole carbon source. To probe function of Scol678, the SCO1678 gene was overexpressed on a moderate copy number plasmid pKC1139 and in-frame deletion mutant $S$. coelicolor $\Delta \mathrm{gntR}$ was generated. Both strains as well as the wild type were grown in liquid SMM or on MM-agar plates supplemented with either glucose or gluconate $(1 \%$, w/v). No differences in growth rate and sporulation were detected among the strains (Figure 2). Complementation of $\Delta \mathrm{gntR}$ with SCO1678 (plasmid pSET-gntR) also had no recognizable effects on growth. Our results agree with the assumption that SCO1678 encodes a repressor of the gnt genes. We noted, though, that $\triangle \mathrm{gntR}$ on gluconate-containing medium had different coloration, probably because of changes in secondary metabolism, as discussed below (see Section 3.7).

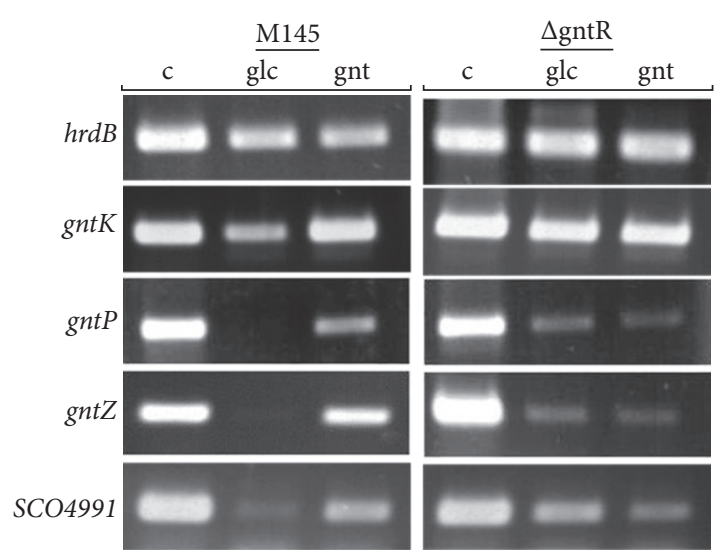

(a)

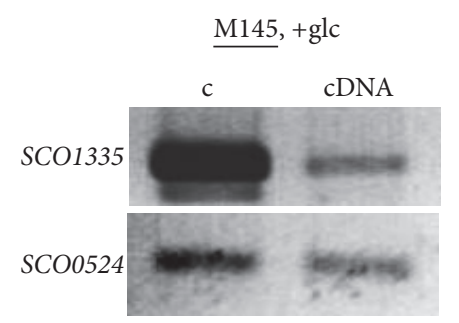

(b)

FIgURE 3: Transcriptional profile of (a) gnt genes in S. coelicolor M145 (M145) and $\Delta$ gntR ( $\triangle$ gntR), and (b) SCO1335 and SCO0524 genes possibly related to pentose phosphate pathway. As a template for RT-PCR chromosomal DNA (c) and cDNA obtained from strains grown in either glucose (glc) or gluconate- (gnt-) containing SMM were used.

3.3. Transcriptional Profile of gnt Genes. To further elucidate SCO1678 function, expression of the gnt operon of $S$. coelicolor was investigated by RT-PCR. For this purpose, a wild type strain and $S$. coelicolor $\Delta$ gntR were grown in liquid SMM medium supplemented with either glucose or gluconate as a sole carbon source. Data are summarized in Figure 3. We note here that our analysis was not quantitative, and transcription level can only be roughly compared within 
one strain. Transcription of $g n t K$, gntP, gntZ, and SCO4991 in wild type strain M145 when cultured in gluconate-containing medium indicates their involvement in gluconate metabolism and transport. However, transcription of gntK and SCO4991 was also detected in M145 in presence of glucose, although their level was lower comparing to that in presence of gluconate. Further analysis of expression of gnt gene in $S$. coelicolor $\Delta$ gntR revealed that in presence of either tested sugar, all four gene transcriptions were detectable.

Absence of $g n t P, g n t Z$ transcription, and reduced level of $g n t K$ transcription in presence of glucose and transcription of all four genes in the presence of gluconate implied that Sco1678 is a gluconate-dependent repressor for the transcription of gnt genes. Transcription of gntK and SCO4991 in the presence of glucose was puzzling. However, glucose can be converted into gluconate and gluconate-6-phosphate and further metabolized through the pentose phosphate pathway. In this case two enzymes are involved: glucose1-dehydrogenase (Sco1335) converts glucose to glucono-1,5lactone which is further metabolized to gluconate by gluconolactonase (Sco0524). These same molecules may induce gnt operon to some extent. Both aforementioned genes were expressed during wild type growth in glucose-containing SMM (Figure 3(b)). Transcription of SCO4991 in presence of either carbohydrate implies that this transporter can be involved in uptake of not only gluconate but other sugars as well.

3.4. Binding of Recombinant Scol678 Protein to Promoter Regions of Putative Target Genes. Pure hexahistidine-tagged Scol678 (GntR-His; 0.93 to $4.14 \mathrm{pmol}$ ) was incubated with Cy5-labeled DNA fragments encompassing promoters of $g n t R-g n t K$, gntP, and $g n t Z$, and reaction products were separated in native acrylamide gel. As shown in Figure 4, GntR-His shifts gntR-gntK intergeneric region starting from a concentration of $0.93 \mathrm{pmol}$. No band shifts were observed with promoters of gntP and gntZ even when higher concentrations of the protein were applied. This agrees with our RT-PCR analysis of gnt operon (see Figure 1(b)), showing that $g n t K, g n t P$, and $g n t Z$ are transcribed as a polycistronic mRNA. Therefore, Scol678 binds only to gntR-gntK region.

3.5. Identification of Sco1678 Effector Molecules. GntR type transcriptional factors act as repressors of gene transcription. Upon binding the appropriate effector molecule, the GntR repressors are no longer able to recognize promoters [23]. In most cases, such an effector molecule is a metabolite from the pathway where products of target genes are involved. To identify the putative effector molecule of GntR the DNA-binding shift assay was performed in the presence of gluconate and gluconolactone as well as glucose (the latter was used as a negative control). For this purpose, purified GntR-His was incubated with $g n t R-K$ intergeneric region in binding buffer that contains putative ligand $(5 \mathrm{mM})$ and subsequently separated in a native acrylamide gel. As shown in Figure 5 gluconate and gluconolactone interfered with binding of GntR-His to the $g n t R-K$ region but could not release the DNA completely. The presence of glucose did not affect protein-DNA interaction.

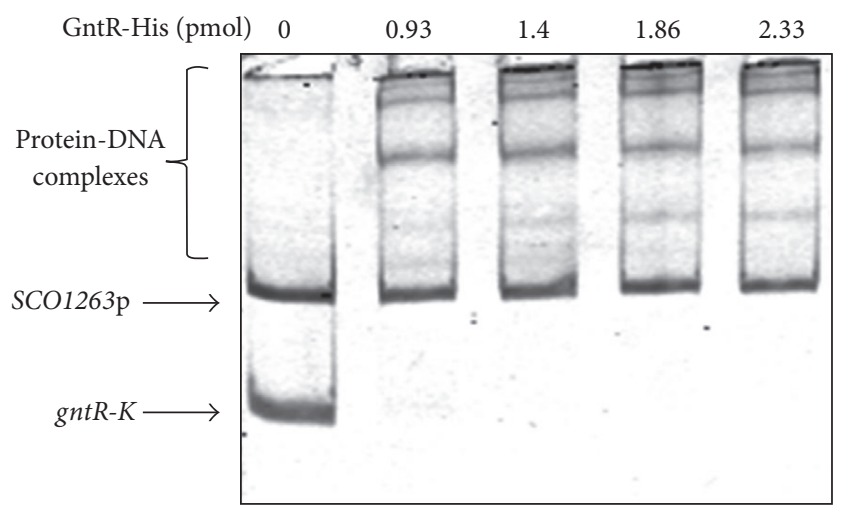

(a)

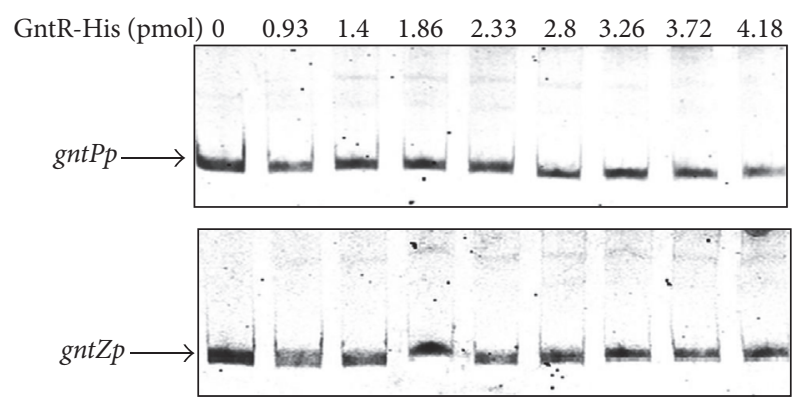

(b)

Figure 4: Binding of Scol678 (GntR) to gntR-K region (a) and to promoters of gntP and $g n t Z$ (b). 0.2 pmol DNA fragment was incubated with indicated GntR-His concentration. Promoter region of SCO1263 was used in reaction as a negative control to check GntRHis specificity to its target $(g n t)$ genes.

3.6. Autoregulatory Function of SCO1678. Most GntR regulators either repress or activate the transcription of their own genes. To explore this further, promoter of SCO1678 was fused with $g u s A$ reporter gene encoding $\beta$-glucuronidase, and the resulting construct was introduced into both $S$. coelicolor $\Delta$ gntR and wild type strains. Data are summarized in Figure 6. Fivefold increased SCO1678 transcription was observed in the wild type grown in SMM-glucose medium as compared to those grown in SMM-gluconate medium. No differences in transcription were observed for SCO1678 deletion mutant grown in presence of either glucose or gluconate, and it was equal to the transcription level observed for the wild type grown on gluconate. Our data showed that Sco1678 upregulates its own gene transcription and that the SCO1678 promoter is not activated in the presence of the effector molecule gluconate.

3.7. S. coelicolor $\Delta$ gntR Produces Yellow Polyketide Coelimycin. $S$. coelicolor produces prolifically at least five natural compounds: ACT, RED, methylenomycin, calcium-dependent antibiotic, and coelimycin [24]. Secondary metabolite production by Streptomyces is tightly linked to physiological and nutritional status. To investigate putative influence of SCO1678 deletion on ACT and RED production, strains were grown in YMPG, R2YE, and SMM media supplemented with either glucose or gluconate. No differences in antibiotics 


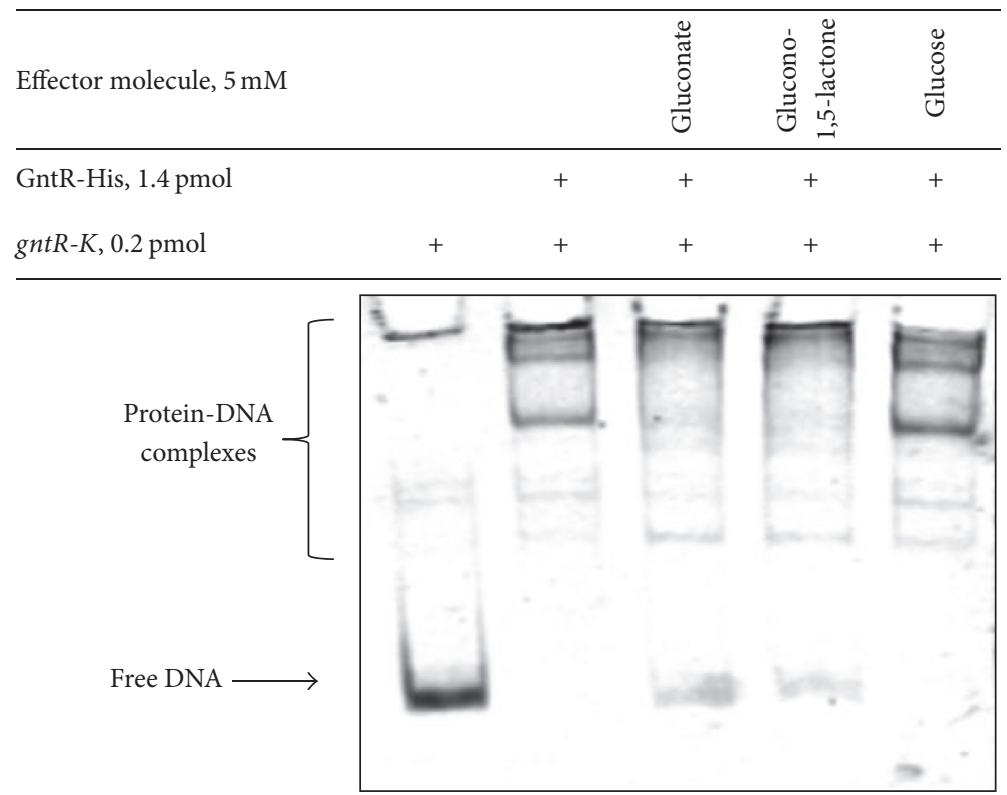

FiguRE 5: EMSA-mediated identification of putative effector molecules for recombinant Sco1678 protein. Binding of Scol678 (1.4 pM) to $g n t R-K$ intergenic region was tested in presence of effectors mentioned in the figure. See Materials and Methods for workup conditions.

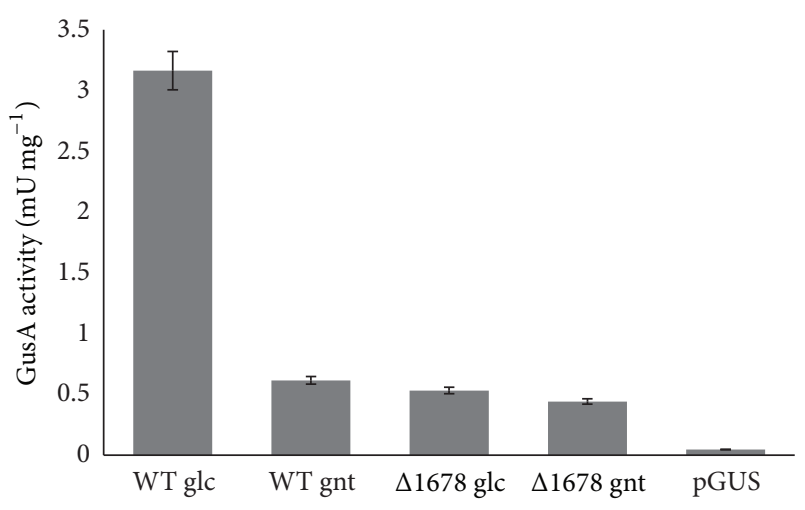

FIGURE 6: Transcriptional activity of SCO1678 (gntR) promoter in S. coelicolor WT (M145) and $\Delta$ gntR. The $\beta$-glucuronidase activity was measured from both strains carrying pGUS-gntR grown for 36 hours in SMM medium supplemented with glucose (WT glc, $\Delta 1678 \mathrm{glc}$ ) or gluconate (WT gnt, $\Delta 1678 \mathrm{gnt}$ ). S. coelicolor pGUS was grown in SMM with glucose and used as a control strain. Data represent mean values of three independent replicates. Error bars, \pm 2 SD.

production were observed in YMPG and R2YE media in comparison to the wild type. In glucose-containing SMM medium both strains produced ACT (Figure 7(a)). However, the level of its production was decreased in the presence of gluconate. Moreover, gluconate triggered the production of a yellow compound, most likely coelimycin (CPK), in S. coelicolor $\Delta$ gntR. Yellow pigment accumulation was also observed when strains were grown on gluconate-containing Oxoid agar plates (Figures 7(b) and 7(c)). Secondary metabolism profile (coloration of agar plates) reverted to wild type when SCO1678 was introduced (on integrative plasmid pSET-gntR) into $\Delta$ gntR (data not shown). The identity of yellow pigment to CPK was established via analytical HPLC-MS analysis.
The new peak (absent in extracts of M145) showed identical retention time (16.5 min) with standard Coelimycin P1, and based on the high resolution and high accurate MS data $\left(349.1214 \mathrm{Da} ;(\mathrm{M}+\mathrm{H})^{+}\right)$, identical molecular formulae are generated as coelimycin P1; UV absorbance spectra of our peak and CPK were also the same.

\section{Discussion}

Streptomycetes can metabolize a variety of carbohydrates including gluconic acid. In this work, we address for the first time the genetic basis of the ability of model strain, S. coelicolor, to utilize gluconate. First, it is imported into the cell by gluconate permease (GntP = Sco1680, maybe Sco4991), after phosphorylation to gluconate-6-phosphate by gluconokinase (GntK = Sco1679) and it then enters the pentose phosphate pathway. Typically, genes responsible for gluconate uptake are organized into an operon under negative control of the GntR repressor [25] (see Figure 1). Transcription level of key gnt genes of $S$. coelicolor were high when grown in gluconatecontaining medium and were absent or severely repressed when grown in the presence of glucose. These observations point to involvement of respective proteins in gluconate metabolism. By combining the results of SCO1678 knockout, RT-PCR of gnt operon, and EMSA of Sco1678 protein, we can safely conclude that Sco1678 is repressor of gnt operon.

Sco1678 binds to promoter region of gluconokinase, but not permease or dehydrogenase, suggesting that these genes are transcribed as polycistronic mRNA. This hypothesis was confirmed by RT-PCR because we could amplify gntK-P and gntP-Z intergenic regions from cDNA (Figure 1(b)).

In SMM medium gluconate inhibits ACT production in both strains. This is not the first case where gluconate has abolished antibiotic production. For instance, cocultivation 


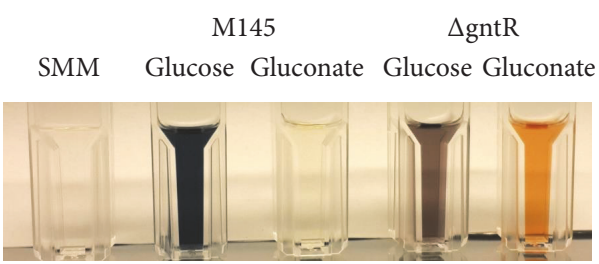

(a)

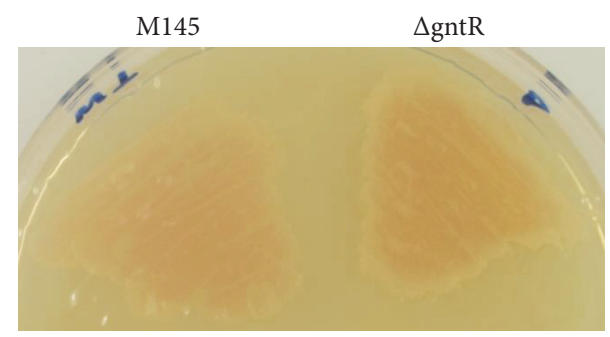

(b)

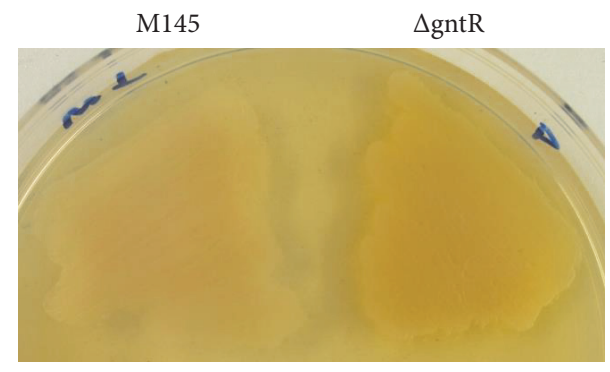

(c)

FIGURE 7: Gluconate inhibits ACT production in S. coelicolor M145 and triggers production of yellow-pigmented compound, $\mathrm{CPK}$, in $S$. coelicolor $\Delta$ gntR. Spent media in cuvettes are shown (a) where respective strains were grown. The production levels correspond to equal amounts of biomass, as judged by Bradford protein assay. On glucose-containing SMM agar both M145 and $\Delta$ gntR produce ACT, and yellow pigment was not obvious (b), while on gluconate-containing Oxoid agar (c) these strains differ in secondary metabolism profile.

of S. coelicolor and Pseudomonas fluorescens BBc6R8, a producer of gluconic acid, stops ACT production [26]. Gluconate inhibits prodigiosin biosynthesis in Serratia sp. ATCC39006. In this case PigT regulator activates transcription of the biosynthetic operon pigA-O, but addition of gluconate decreases transcription of the latter. PigT shows high level homology to E. coli GntR protein [27]. Our working hypothesis is that gluconate, in the absence of the other carbon sources and regulatory function of Scol678, serves as a metabolic signal that switches secondary metabolism from production of typical metabolites (ACT and RED) to minor or cryptic ones, such as coelimycin. We speculate that this secondary metabolic switch is mediated by a regulatory protein not yet known. Work is currently underway in our laboratories to experimentally explore this hypothesis.

\section{Conclusions}

Protein Sco1678, encoded within Streptomyces coelicolor M145 genome, belongs to twelve of the most conserved regulators of GntR family across class of Actinobacteria. Here we show for the first time that SCO1678 gene encodes GntR, or repressor of gluconate utilization operon. Its repressor function is exerted via binding to single promoter upstream of gluconokinase gene SCO1679 and is responsive to gluconate. We revealed that GntR in S. coelicolor constitutes a regulatory checkpoint for secondary metabolism, because under certain growth conditions the SCO1678 knockout decreases actinorhodin titers and induces the production of otherwise cryptic polyketide coelimycin. Our data suggest that further studies of SCO1678 (gntR) are worth pursuing. They will lead to further insight into coordination of primary and secondary metabolic pathways and help devise novel approaches towards the induction of silent gene clusters in actinomycetes.

\section{Disclosure}

Present address of Olga Tsypik and Roman Makitrynskyy is Albert-Ludwigs-University of Freiburg, Pharmazeutische Biologie, 79104 Freiburg, Germany.

\section{Conflicts of Interest}

The authors declare that there are no conflicts of interest regarding the publication of this paper.

\section{Acknowledgments}

The authors thank Kaitlyn Pinkett for proofreading their manuscript. Olga Tsypik was supported by DAAD fellowship. Research groups of Bohdan Ostash and Victor Fedorenko are supported by Grants BG-41Nr and BG-46F, respectively, from the Ministry of Education and Science of Ukraine.

\section{References}

[1] E. A. Barka, P. Vatsa, L. Sanchez et al., "Taxonomy, physiology, and natural products of actinobacteria," Microbiology and Molecular Biology Reviews, vol. 80, no. 1, pp. 1-43, 2016.

[2] M. B. Jones, W. C. Nierman, Y. Shan et al., "Reducing the bottleneck in discovery of novel antibiotics," Microbial Ecology, vol. 73, no. 3, pp. 658-667, 2017.

[3] G. R. Lewin, C. Carlos, M. G. Chevrette et al., "Evolution and ecology of actinobacteria and their bioenergy applications," Annual Review of Microbiology, vol. 70, no. 1, pp. 235-254, 2016.

[4] T. Rey and B. Dumas, "Plenty is no plague: Streptomyces symbiosis with crops," Trends in Plant Science, vol. 22, no. 1, pp. 3037, 2017.

[5] G. Liu, K. F. Chater, G. Chandra, G. Niu, and H. Tan, "Molecular regulation of antibiotic biosynthesis in Streptomyces," Microbiology and Molecular Biology Reviews, vol. 77, no. 1, pp. 112-143, 2013.

[6] A. J. Book, G. R. Lewin, B. R. McDonald et al., "Evolution of high cellulolytic activity in symbiotic Streptomyces through selection of expanded gene content and coordinated gene expression," PLoS Biology, vol. 14, no. 6, Article ID e1002475, 2016.

[7] J. M. He, H. Zhu, G. S. Zheng et al., "Direct involvement of the master nitrogen metabolism regulator $\mathrm{GlnR}$ in antibiotic 
biosynthesis in Streptomyces," The Journal of Biological Chemistry, vol. 291, pp. 26443-26454, 2016.

[8] J. F. Martín and P. Liras, "Cascades and networks of regulatory genes that control antibiotic biosynthesis," Sub-Cellular Biochemistry, vol. 64, pp. 115-138, 2012.

[9] O. Tsypik, O. Yushchuk, N. Zaburannyi et al., “Transcriptional regulators of GntR family in Streptomyces coelicolor A3(2): analysis in silico and in vivo of YtrA subfamily," Folia Microbiologica, vol. 61, no. 3, pp. 209-220, 2016.

[10] J. Persson, K. F. Chater, and K. Flärdh, "Molecular and cytological analysis of the expression of Streptomyces sporulation regulatory gene whiH," FEMS Microbiology Letters, vol. 341, no. 2, pp. 96-105, 2013.

[11] M. J. Bush, N. Tschowri, S. Schlimpert, K. Flärdh, and M. J. Buttner, "C-di-GMP signalling and the regulation of developmental transitions in streptomycetes," Nature Reviews Microbiology, vol. 13, no. 12, pp. 749-760, 2015.

[12] M. Urem, M. A. Światek-Połatyńska, S. Rigali, and G. P. van Wezel, "Intertwining nutrient-sensory networks and the control of antibiotic production in Streptomyces," Molecular Microbiology, vol. 102, no. 2, pp. 183-195, 2016.

[13] B. Gust, G. Chandra, D. Jakimowicz, T. Yuqing, C. J. Bruton, and K. F. Chater, " $\lambda$ red-mediated genetic manipulation of antibiotic-producing Streptomyces," Advances in Applied Microbiology, vol. 54, pp. 107-128, 2004.

[14] J. Sambrook, E. F. Fritsch, and T. Maniatis, Molecular Cloning. A Laboratory Manual, Cold Spring Harbor Laboratory Press, Cold Spring Harbor, NY, USA, 1989.

[15] T. Kieser, M. J. Bibb, M. J. Buttner, K. F. Chater, and D. A. Hopwood, Practical Streptomyces Genetics, John Innes Foundation, Norwich, UK, 2000.

[16] K. Pawlik, M. Kotowska, and P. Kolesiński, "Streptomyces coelicolor A3(2) produces a new yellow pigment associated with the polyketide synthase Cpk," Journal of Molecular Microbiology and Biotechnology, vol. 19, no. 3, pp. 147-151, 2010.

[17] R. Makitrynskyy, B. Ostash, O. Tsypik et al., "Pleiotropic regulatory genes $b l d A, a d p A$ and $a b s B$ are implicated in production of phosphoglycolipid antibiotic moenomycin," Open Biology, vol. 3, no. 10, Article ID 130121, 2013.

[18] M. Fedoryshyn, E. Welle, A. Bechthold, and A. Luzhetskyy, "Functional expression of the Cre recombinase in actinomycetes," Applied Microbiology and Biotechnology, vol. 78, no. 6, pp. 1065-1070, 2008.

[19] Y. Tiffert, P. Supra, R. Wurm, W. Wohlleben, R. Wagner, and J. Reuther, "The Streptomyces coelicolor GlnR regulon: identification of new GlnR targets and evidence for a central role of GlnR in nitrogen metabolism in actinomycetes," Molecular Microbiology, vol. 67, no. 4, pp. 861-880, 2008.

[20] Z. Zhou, N. Sun, S. Wu, Y.-Q. Li, and Y. Wang, "Genomic data mining reveals a rich repertoire of transport proteins in Streptomyces," BMC Genomics, vol. 17, article 510, 2016.

[21] S. Tong, A. Porco, T. Isturiz, and T. Conway, "Cloning and molecular genetic characterization of the Escherichia coli gntR, $g n t K$, and gnt $U$ genes of GntI, the main system for gluconate metabolism," Journal of Bacteriology, vol. 178, no. 11, pp. 32603269, 1996.

[22] Y. Fujita and Y. Miwa, "Identification of an operator sequence for the Bacillus subtilis gnt operon," Journal of Biological Chemistry, vol. 264, no. 7, pp. 4201-4206, 1989.

[23] P. A. Hoskisson and S. Rigali, "Variation in form and function the helix-turn-helix regulators of the GntR superfamily," in Advances in Applied Microbiology, vol. 69, chapter 1, pp. 1-22, Elsevier, 2009.

[24] Y. Jeong, J.-N. Kim, M. W. Kim et al., "The dynamic transcriptional and translational landscape of the model antibiotic producer Streptomyces coelicolor A3(2)," Nature Communications, vol. 7, Article ID 11605, 2016.

[25] D. J. Haydon and J. R. Guest, "A new family of bacterial regulatory proteins," FEMS Microbiology Letters, vol. 79, no. 2-3, pp. 291-295, 1991.

[26] J. Galet, A. Deveau, L. Hôtel, P. Leblond, P. Frey-Klett, and B. Aigle, "Gluconic acid-producing Pseudomonas sp. prevent $\gamma$-actinorhodin biosynthesis by Streptomyces coelicolor A3(2)," Archives of Microbiology, vol. 196, no. 9, pp. 619-627, 2014.

[27] P. C. Fineran, L. Everson, H. Slater, and G. P. C. Salmond, "A GntR family transcriptional regulator (PigT) controls gluconate-mediated repression and defines a new, independent pathway for regulation of the tripyrrole antibiotic, prodigiosin, in Serratia," Microbiology, vol. 151, no. 12, pp. 3833-3845, 2005. 

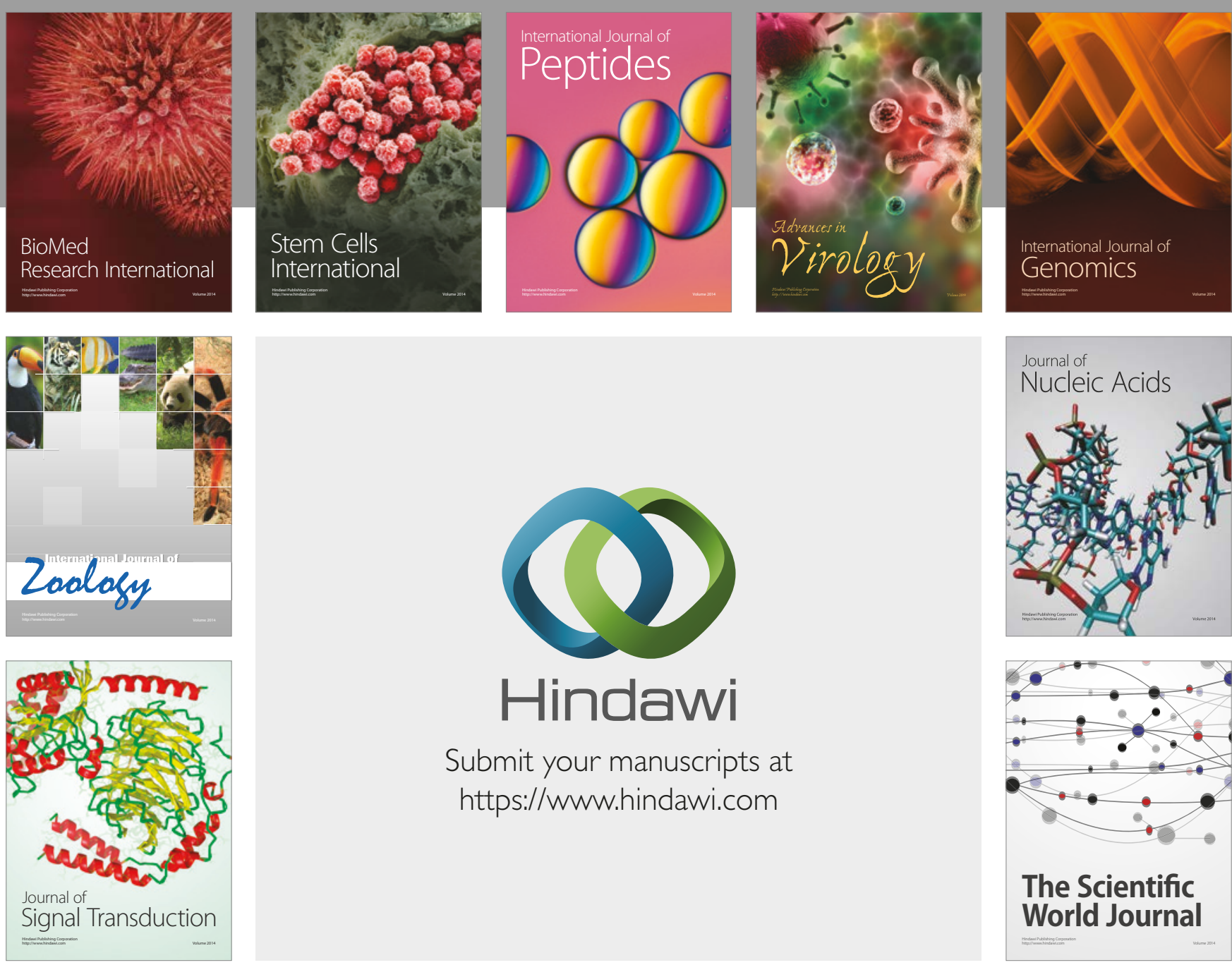

Submit your manuscripts at

https://www.hindawi.com
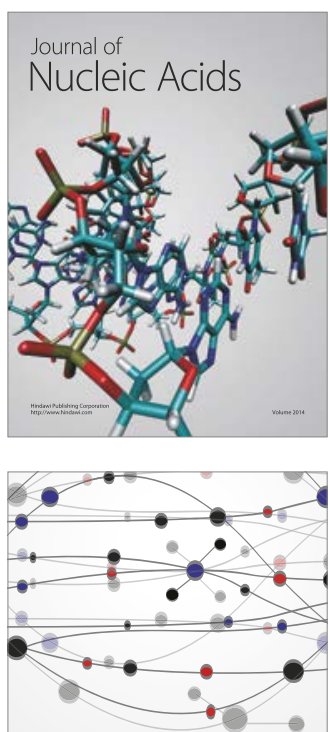

The Scientific World Journal

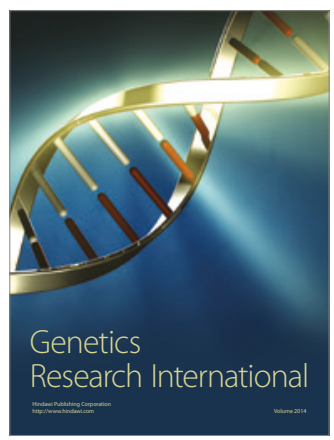

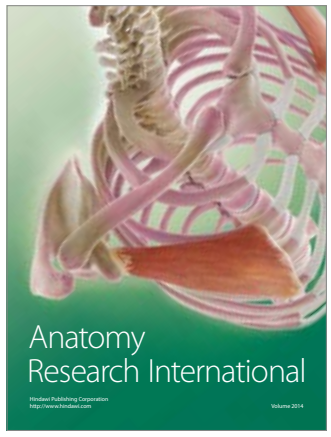

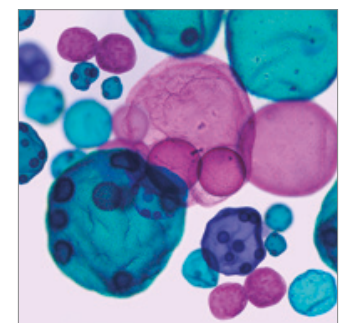

International Journal of Microbiology
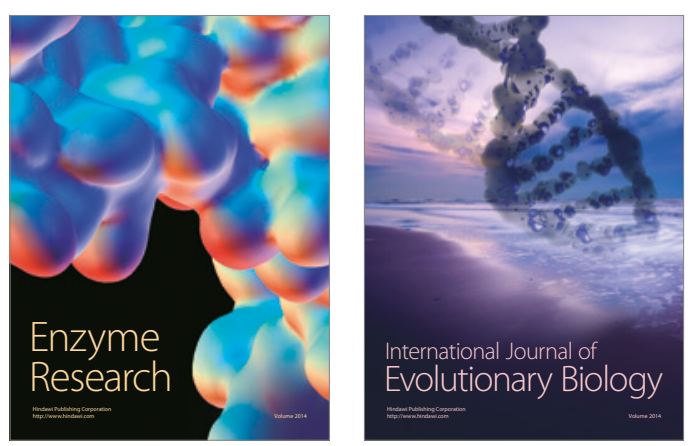
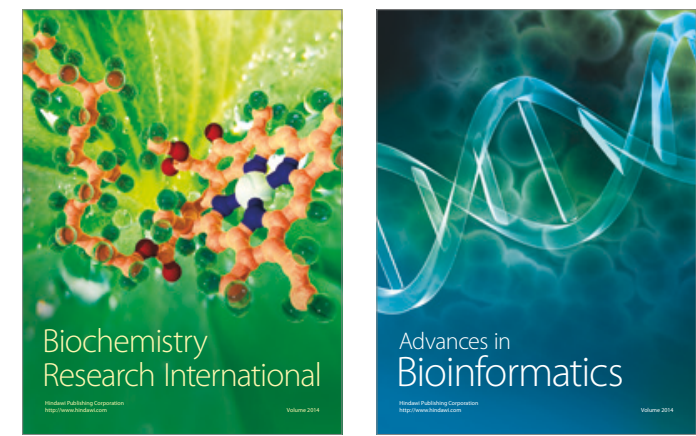

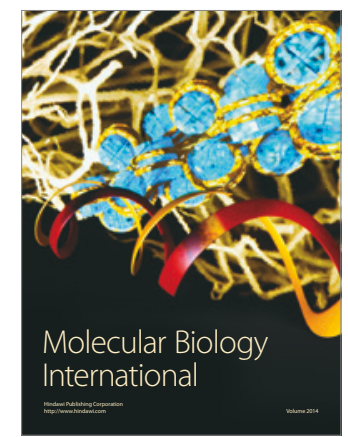

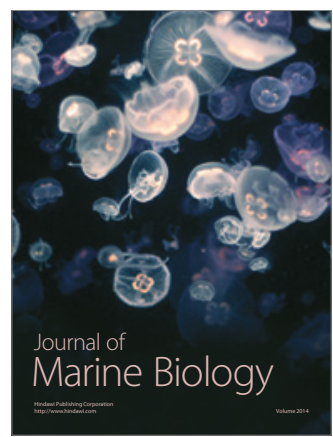

